\title{
MONOTONE ECONOMICAL SCHEMES FOR QUASILINEAR PARABOLIC EQUATIONS
}

\author{
N.V. DZENISENKO ${ }^{1}$, A.P. MATUS ${ }^{2}$ and P.P. MATUS ${ }^{3}$ \\ 1 Belarusian State Economic University \\ 26 Partyzanskii Avenue, 220672, Minsk-70, Belarus \\ 2 ROSTEC Inc \\ 8295 County Road 10, Corcoran, MN 55357 USA \\ E-mail: aleh@rostec.net \\ 3 Institute of Mathematics of NAS of Belarus \\ 11 Surganov Str., 220072 Minsk, Belarus \\ E-mail: matus@im.bas-net.by
}

Received September 17, 2002

\begin{abstract}
In order to approximate a multidimensional quasilinear parabolic equation with unlimited nonlinearity the economical vector-additive scheme is constructed. It is shown that its solution satisfies the maximum principle and, hence, the scheme is monotone. The proof is based on the equivalence of the vector-additive scheme and the scheme of summarized approximation (locally one-dimensional scheme). The a priori estimates of the difference solution in the uniform norm are obtained.
\end{abstract}

Key words: finite-difference schemes, nonlinear parabolic problems, maximum principle.

\section{INTRODUCTION}

In order to obtain an approximate solution of multidimensional nonstationary problems of mathematical physics the different classes of additive schemes (operator-splitting schemes) are widely used [4; 5]. In a number of papers $[1 ; 7]$, the vector-additive schemes (multicomponent schemes of alternating directions) are considered. These schemes are of full approximation.

In view of stability, the most definite results were obtained for linear problems by the study of the difference schemes in Hilbert grid spaces. It also concerns the stability and convergence of additive operator-difference schemes 
of full approximation. For many problems the issue of the scheme correctness in the uniform norm is fundamental (in the Banach grid space $L_{\infty}$ ). As an example, we may note the problems with unlimited nonlinearity. Stability in the grid norm $C$ for the simplest vector-additive schemes approximating linear multidimensional parabolic equations were considered in the works $[2 ; 6 ; 9]$. The proof is based on the use of the maximum principle and the equivalence of the vector-additive schemes and the schemes of summarized approximation. Difference schemes satisfying the maximum principle are called monotone. It is very important to save the monotonicity property of difference schemes for numerical solution of applied problems with the aid of a computer.

The problems with unlimited nonlinearity are characterized by the fact that specific properties imposed on solution-dependent coefficients of the equation are satisfied only in a range of values of the exact solution or in its small neighborhood. Thus, for these properties to hold true for coefficients of the difference scheme, it is necessary to prove that the approximate solution lies in the range of values of the exact solution. This circumstance requires the study of the difference solution properties in the norm $C$.

In this paper, monotonicity of the economical vector-additive scheme of full approximation for multidimensional quasilinear parabolic equations with nonlinearities grown with no limit is proved. To obtain the a priori estimates in the norm $C$, the maximum principle and the equivalence of the multicomponent method of alternating directions and the schemes of summarized approximation are used.

\section{MAXIMUM PRINCIPLE AND ITS COROLLARIES}

In this section, we give the maximum principle and its corollaries, which will be used to prove that the difference scheme solution lies in the range of values of the exact solution or in its small neighborhood. We will also obtain the a priori estimates for solutions of the difference schemes approximating the nonlinear parabolic equations with nonlinearities grown with no limit.

We use the following canonical form [4] of a difference scheme:

$$
A(x) y(x)=\sum_{\xi \in \mathcal{M}^{\prime}(x)} B(x, \xi) y(\xi)+F(x), x \in \omega_{h}, y(x)=\mu(x), x \in \gamma_{h},
$$

where $A(x), B(x, \xi), F(x), \mu(x)$ are given grid functions, $\mathcal{M}^{\prime}(x)=\mathcal{M}(x) \backslash\{x\}$, $\mathcal{M}(x)$ is a stencil of the scheme, $y(x)$ is a discrete solution, $\omega_{h}$ is a set of the boundary nodes. Let us formulate the maximum principle in the following way.

Theorem 2.1 [4]. Let the following conditions of the coefficients positivity 
be satisfied for equation (2.1):

$$
A(x)>0, \quad B(x, \xi)>0, D(x)=A(x)-\sum_{\xi \in \mathcal{M}^{\prime}(x)} B(x, \xi) \geq 0, x \in \omega_{h},
$$

and the following inequalities be valid:

$$
F(x) \geq 0, x \in \omega_{h}, \mu(x) \geq 0, x \in \gamma_{h} .
$$

Then the function $y(x)$ is nonnegative, i.e., $y(x) \geq 0, x \in \bar{\omega}_{h}$. If

$$
F(x) \leq 0\left(x \in \omega_{h}\right), \quad \mu(x) \leq 0\left(x \in \gamma_{h}\right),
$$

then $y(x) \leq 0$ for all $x \in \bar{\omega}_{h}$.

Corollary 2.1. Let conditions (2.2) be satisfied and

$$
\begin{gathered}
F(x) \geq u_{1}, x \in \omega_{h}, \mu(x) \geq u_{1}, x \in \gamma_{h}, u_{1}=\text { const } \geq 0, \\
0 \leq D \leq 1 .
\end{gathered}
$$

Then the following inequality is valid:

$$
y(x) \geq u_{1}, x \in \bar{\omega}_{h}, \quad \bar{\omega}_{h}=\omega_{h} \cup \gamma_{h} .
$$

Proof. In fact, substituting $y=\tilde{y}+u_{1}$ into equation (2.1), we get the problem for $\tilde{y}$

$$
A(x) \tilde{y}(x)=\sum_{\xi \in \mathcal{M}^{\prime}(x)} B(x, \xi) \tilde{y}(\xi)+\tilde{F}(x), x \in \omega_{h}, \tilde{y}(x)=\tilde{\mu}(x), x \in \gamma_{h} .
$$

In the strength of conditions $(2.2)-(2.4)$,

$$
\begin{gathered}
\tilde{F}(x)=F(x)-u_{1} D(x) \geq(1-D) u_{1} \geq 0, \quad x \in \omega_{h}, \\
\tilde{\mu}(x)=\mu(x)-u_{1} \geq 0, \quad x \in \gamma_{h} .
\end{gathered}
$$

Applying Theorem 2.1 to problem (2.7), (2.8) we find that $\tilde{y}(x) \geq 0$ for all $x \in \bar{\omega}_{h}$. Hence, $y=\tilde{y}+u_{1} \geq 0$.

Let us define the grid norms

$$
\begin{aligned}
& \|\cdot\|_{\bar{C}}=\max _{x \in \bar{\omega}_{h}}|\cdot|, \\
& \|\cdot\|_{C_{\gamma}}=\max _{x \in \gamma_{h}}|\cdot|,\|\cdot\|_{C}=\max _{x \in \omega_{h}}|\cdot| .
\end{aligned}
$$


Corollary 2.2 [4]. Let the stronger constraints on the coefficients be valid:

$$
A(x)>0, B(x, \xi)>0, D(x)=A(x)-\sum_{\xi \in \mathcal{M}^{\prime}(x)} B(x, \xi)>0, x \in \omega_{h} .
$$

Then for the solution of problem (2.1) the following estimate is true:

$$
\|y\|_{\bar{C}} \leq \max \left\{\|y\|_{C_{\gamma}},\|F / D\|_{C}\right\}
$$

\section{STATEMENT OF THE DIFFERENTIAL PROBLEM}

In this section, we formulate the initial boundary-value problem for the multidimensional quasilinear parabolic equation and indicate the intrinsic properties of problems with unlimited nonlinearity.

In the cylindrical domain $\bar{Q}_{T}=\{(x, t): x \in \bar{\Omega}, 0 \leq t \leq T\}, x=$ $\left(x_{1}, x_{2}, \ldots, x_{p}\right), \bar{\Omega}=\left\{0 \leq x_{\alpha} \leq l_{\alpha}, \alpha=1,2, \ldots, p\right\}, \bar{\Omega}=\bar{\Omega} \cup \gamma$, where $\gamma$ is the boundary, we consider the first boundary-value problem for $p$-dimensional heat conduction equation

$$
\begin{gathered}
\frac{\partial u}{\partial t}=\sum_{\alpha=1}^{p} \frac{\partial}{\partial x_{\alpha}}\left(k_{\alpha}(x, t, u) \frac{\partial u}{\partial x_{\alpha}}\right)+f(x, t), f(x, t) \geq 0,(x, t) \in Q_{T} \\
u(x, 0)=u_{0}(x), x \in \bar{\Omega}, u(x, t)=\mu(x, t), x \in \gamma, t>0 .
\end{gathered}
$$

Let us define the range of values of the exact solution $\bar{D}_{u}$ by the inequalities

$$
\begin{gathered}
0<u_{1} \leq u(x, t) \leq \max \left\{\max _{t}\|\mu\|_{C_{\gamma}},\left\|u_{0}\right\|_{C(\bar{\Omega})}\right\}+\int_{0}^{t}\|f(\xi)\|_{C(\bar{\Omega})} d \xi \leq u_{2} \\
(x, t) \in \bar{Q}_{T}, \quad f(x, t) \geq 0
\end{gathered}
$$

We set up a neighborhood of the exact solution $\bar{D}_{\tilde{u}}$ (which might be quite small):

$$
\left\{1 \leq \tilde{u} \leq u_{2}+r, \quad r=\tau T\left\|\frac{\partial f}{\partial t}\right\|_{C\left(\bar{Q}_{T}\right)} \rightarrow 0 \text { for } \tau \rightarrow 0\right\}
$$

The intrinsic feature of problems with unlimited nonlinearity is that the basic properties (positiveness, boundedness, etc.) of the nonlinear coefficients $k_{\alpha}(x, t, u), \quad \alpha=1,2, \ldots, p$ are not satisfied for all the values $u$ as a parameter, but only for $u$ in the range of values of the exact solution $\bar{D}_{u}$ or its small neighborhood. Further we assume that

$$
0<k_{1} \leq k_{\alpha}(x, t, u) \leq k_{2}, \quad \alpha=1,2, \ldots, p, \quad(x, t) \in \bar{Q}_{T}, \quad u \in \bar{D}_{\tilde{u}} .
$$


For example, for problems of radiant heat conductivity the exponential nonlinearity is typical: $k_{\alpha}(x, t, u)=u^{\sigma}, \quad \alpha=1,2, \ldots, p, \quad \sigma=$ const. The positivity condition (3.3) is fulfilled for these coefficients provided that $u>0$ only. In the other example, $k_{\alpha}(x, t, u)=u-\sigma_{\alpha}, \quad \sigma_{\alpha}=$ const $, \quad \alpha=1,2, \ldots, p$, this condition is satisfied for $u>\sigma_{\alpha}$.

\section{DIFFERENCE SCHEME}

In this section, on the basis of the stuff given in the papers $[1 ; 2 ; 7 ; 8]$, the economical non-iterative vector-additive scheme of full approximation with different numerical representations of the initial data is constructed.

In the rectangle $\bar{\Omega}$ we introduce the uniform grid $\bar{\omega}=\bar{\omega}_{h} \times \bar{\omega}_{\tau}$,

$$
\bar{\omega}_{\tau}=\left\{t_{n}=n \tau, \quad n=0,1, \ldots\right\}, \quad \bar{\omega}_{h}=\omega_{h} \cup \gamma_{h},
$$

where a set of the inner nodes is defined by

$$
\begin{aligned}
& \omega_{h}=\left\{x_{i}=\left(x_{1}^{\left(i_{1}\right)}, x_{2}^{\left(i_{2}\right)}, \ldots, x_{p}^{\left(i_{p}\right)}\right), \quad x_{\alpha}^{\left(i_{\alpha}\right)}=i_{\alpha} h_{\alpha}, i_{\alpha}=1,2, \ldots, N_{\alpha}-1,\right. \\
& \left.h_{\alpha} N_{\alpha}=l_{\alpha}, \quad \alpha=1,2, \ldots, p\right\}
\end{aligned}
$$

and $\gamma_{h}$ is a set of the boundary nodes. The following difference scheme approximates differential problem (3.1), (3.2) in $\bar{\omega}$ :

$$
\begin{aligned}
& y_{1 t}=\Lambda_{1} \hat{y}_{1}+\sum_{m=2}^{p} \Lambda_{m} y_{m}+\hat{\varphi}, \\
& y_{2 t}=\Lambda_{1} \hat{y}_{1}+\hat{\Lambda}_{2} \hat{y}_{2}+\sum_{m=3}^{p} \Lambda_{m} y_{m}+\hat{\varphi}, \\
& \ldots \ldots \ldots \ldots \ldots \ldots \ldots \cdots \\
& y_{p t}=\Lambda_{1} \hat{y}_{1}+\sum_{m=2}^{p} \hat{\Lambda}_{m} \hat{y}_{m}+\hat{\varphi}, \\
& \left.y_{\alpha}\right|_{\gamma_{h}}=\mu(x, t), \quad x \in \gamma_{h}, \quad t \in \omega_{\tau},
\end{aligned}
$$

with two kinds of the initial data approximation:

$$
\begin{aligned}
& \left(E--\tau \Lambda_{\alpha}^{0}\right) y_{\alpha}^{0}=y_{\alpha-1}^{0}, \quad \alpha=2,3, \ldots, p, \quad y_{1}^{0}=u_{0}, \\
& y_{\alpha}(x, 0)=u_{0}(x), \quad \alpha=1,2, \ldots, p, \quad x \in \omega_{h} .
\end{aligned}
$$


Here the following notation has been used:

$$
\begin{aligned}
& y_{\alpha t}=\left(y_{\alpha}\left(x, t_{n+1}\right)-y_{\alpha}\left(x, t_{n}\right)\right) / \tau=\left(\hat{y}_{\alpha}-y_{\alpha}\right) / \tau, \quad x \in \omega_{h}, \\
& \Lambda_{\alpha} y=\left(a_{\alpha} y_{\bar{x}_{\alpha}}\right)_{x_{\alpha}}=\frac{1}{h_{\alpha}}\left(a_{\alpha(+1 \alpha)} y_{x_{\alpha}}-a_{\alpha} y_{\bar{x}_{\alpha}}\right), \\
& v\left( \pm 1_{\alpha}\right)=v\left(x_{1}^{\left(i_{1}\right)}, \ldots, x_{\alpha-1}^{\left(i_{\alpha}-1\right)}, x_{\alpha}^{\left(i_{\alpha}\right)} \pm h_{\alpha}, x_{\alpha+1}^{\left(i_{\alpha}+1\right)}, \ldots, x_{p}^{\left(i_{p}\right)}\right), \\
& y_{x_{\alpha}}=\left(y\left(+1_{\alpha}\right)-y\right) / h_{\alpha}, \quad y_{\bar{x}_{\alpha}}=\left(y-y\left(-1_{\alpha}\right)\right) / h_{\alpha}, \\
& a_{\alpha}=a_{\alpha}^{n}=0.5\left(k_{\alpha}\left(x, t_{n}, y_{1}^{n}\right)+k_{\alpha}\left(x\left(-1_{\alpha}\right), t_{n}, y_{1\left(-1_{\alpha}\right)}^{n}\right)\right), \\
& \Lambda_{\alpha} \hat{y}_{\alpha}=\left(a_{\alpha} \hat{y}_{\bar{x}_{\alpha}}\right)_{x_{\alpha}}, \quad \hat{\Lambda}_{\alpha} \hat{y}_{\alpha}=\left(\hat{a}_{\alpha} \hat{y}_{\bar{x}_{\alpha}}\right)_{x_{\alpha}}, \quad \hat{\varphi}=f(x, \hat{t}) .
\end{aligned}
$$

The numerical solution can be found from difference schemes $(4.1)-(4.6)$ in the following way. According to the given boundary conditions (4.4) and initial data (4.5) or (4.6) the solution at the first time layer $y^{1}=y_{1}^{1}=y_{1}(x, \tau)$ can be computed using (4.1) and the formula of the scalar sweep method with respect to variable $x_{1}$ only. Further, using the auxiliary values $y_{2}^{1}, \ldots, y_{p}^{1}$ found and formulas $(4.2)-(4.6)$, we compute $y^{2}=y_{1}^{2}=y_{1}(x, 2 \tau)$ at the second time layer. Then the procedure stated above is repeated. It is essential that in all the equations for determining the pattern functionals $a_{\alpha}\left(x, t, y_{1}\right),(x, t) \in \omega$, it is used the desired grid solution $y=y_{1}$ only.

\section{THE EQUIVALENCE OF VECTOR-ADDITIVE AND LO- CALLY ONE-DIMENSIONAL SCHEMES}

In this section, we will prove the equivalence of the vector-additive schemes and the schemes of summarized approximation. For linear problems these issues were discussed in papers [2;3].

First of all, we consider difference scheme (4.1) - (4.4) with the initial data approximation of (4.5) type. Subtracting in (4.1) - (4.3) two consecutive equations, we get the following system of equations:

$$
\left(y_{\alpha}-y_{\alpha-1}\right)_{t}=\tau\left(\Lambda_{\alpha} y_{\alpha}\right)_{t}, \quad \alpha=2, \ldots, p
$$

Now we subtract equation (4.3) written in the previous time layer from equation (4.1) and obtain the formula

$$
\left(y_{1}-\check{y}_{p}\right)_{t}=\tau\left(\check{\Lambda} y_{1}\right)_{t}+\tau \varphi_{t}, \quad \check{v}=v(t-\tau)=v\left(t_{n-1}\right)
$$

Performing the numerical integration of the system of difference equations (5.1), (5.2) and taking into account the initial data (4.5), we have the following 
system of algebraic equations:

$$
\begin{aligned}
& \frac{y_{\alpha}^{n}-y_{\alpha-1}^{n}}{\tau}=\Lambda_{\alpha}^{n} y_{\alpha}^{n}, \quad \alpha=2,3, \ldots, p, \quad n \geq 0 \\
& \frac{y_{1}^{n+1}-y_{p}^{n}}{\tau}=\Lambda_{1} y_{1}^{n+1}+\varphi^{n+1}, \quad n \geq 0 .
\end{aligned}
$$

Setting $y_{2}^{n}=y^{n+\frac{1}{p}}, \ldots, y_{p}=y^{n+\frac{p-1}{p}}, y_{1}^{n+1}=y^{n+1}$, we obtain the classical version of the method of summarized approximation. Hence, a vector-additive difference scheme of the form (4.1) - (4.5) can be written as a locally onedimensional one.

Note that if we use the standard approximation of the initial data (4.6) instead of formula (4.5), then we get by the numerical integration of system (5.1), (5.2) the following system of difference equations instead of system (5.3), (5.4):

$$
\begin{gathered}
\frac{y_{\alpha}^{n}-y_{\alpha-1}^{n}}{\tau}=\Lambda_{\alpha}^{n} y_{\alpha}^{n}+C_{\alpha}, \quad \alpha=2,3, \ldots, p, \\
\frac{y_{1}^{n+1}-y_{p}^{n}}{\tau}=\Lambda_{1}^{n} y_{1}^{n+1}+\varphi^{n+1}+C_{1},
\end{gathered}
$$

where $C_{\alpha}$ is given by the formula

$$
C_{1}(x)=-\sum_{\alpha=2}^{p} C_{\alpha}(x), \quad C_{\alpha}(x)=-\Lambda_{\alpha}^{0} u_{0}(x), \quad \alpha=2,3, \ldots, p, x \in \bar{\omega}_{h} .
$$

\section{MONOTONICITY AND THE A PRIORI ESTIMATES}

In this section, we will prove the monotonicity of the vector-additive schemes considered and obtain the a priori estimates in the norm $C$ in the nonlinear case. It should be noted that if $y^{n}=y_{1}^{n} \in \bar{D}_{\tilde{u}}$, i. e.,

$$
0<u_{1} \leq y(x, t) \leq u_{2}+r, \quad(x, t) \in \bar{\omega},
$$

then by virtue of formula (3.3) and by the choice of the pattern functionals $a_{\alpha}, \alpha=\overline{1, p}$, we obtain the inequality

$$
0<k_{1} \leq a_{\alpha}(x, t, y) \leq k_{2}, \quad(x, t) \in \omega, \quad y \in \bar{D}_{\tilde{u}} .
$$

While investigating the properties of difference schemes for nonlinear problems with unlimited nonlinearity, the most difficult issue is to prove very inequalities $(6.1)-(6.2)$. Using the maximum principle and its corollaries given in 
Section 2 we show that the approximate solution lies in the neighborhood of the range of values of the exact solution $\left(y^{n} \in \bar{D}_{\tilde{u}}\right)$.

We rewrite equations $(5.3),(5.4)$ in their canonical form (2.1):

$$
\begin{array}{r}
A_{\alpha}^{n} y_{\alpha}^{n}=\frac{\tau}{h_{\alpha}^{2}}\left(a_{\alpha\left(+1_{\alpha}\right)}^{n} y_{\alpha\left(+1_{\alpha}\right)}^{n}+a_{\alpha}^{n} y_{\alpha\left(-1_{\alpha}\right)}^{n}\right)+F_{\alpha}^{n}, \quad \alpha=\overline{2, p} \\
A_{\alpha}^{n}=1+\frac{\tau}{h_{\alpha}^{2}}\left(a_{\alpha\left(+1_{\alpha}\right)}^{n}+a_{\alpha}^{n}\right), \quad F_{\alpha}^{n}=y_{\alpha-1}^{n}, \quad \alpha=\overline{2, p} \\
A_{1}^{n} y_{1}^{n+1}=\frac{\tau}{h_{1}^{2}}\left(a_{1\left(+1_{1}\right)}^{n} y_{1\left(+1_{1}\right)}^{n+1}+a_{1}^{n} y_{1\left(-1_{1}\right)}^{n}\right)+F_{1}^{n} \\
A_{1}^{n}=1+\frac{\tau}{h_{1}^{2}}\left(a_{1\left(+1_{1}\right)}^{n}+a_{1}^{n}\right), \quad F_{1}^{n}=y_{p}^{n}+\tau \varphi^{n+1} .
\end{array}
$$

Note the initial data

$$
y^{0}=y(x, 0)=y_{1}(x, 0)=u_{0}(x)
$$

and boundary condition

$$
y(x, t)=\mu(x, t), \quad x \in \gamma_{h}
$$

lie in the range of values of the exact solution $\bar{D}_{u}$, i.e., the following inequalities are valid:

$$
0<u_{1} \leq y_{1}^{0}, \quad \mu\left(\gamma_{h}, t\right) \leq u_{2}, \quad x \in \bar{\omega}_{h}, \quad t \in \bar{\omega}_{\tau} .
$$

Since $y_{1}^{0} \in \bar{D}_{u}$, then by virtue of formula (3.3) the inequalities (6.2) are satisfied for $n=0$ and for all $\alpha=1,2, \ldots, p$ : $0<k_{1} \leq a_{\alpha}^{0} \leq k_{2}$. Hence, the conditions of the coefficients positivity (2.2) for schemes (6.3), (6.4) are satisfied for $n=0$ provided that $D_{\alpha}^{0}=1$. Estimate (2.10) and formula (6.3) yield the following estimate:

$$
\left\|y_{p}^{0}\right\|_{\bar{C}} \leq\left\|y_{p-1}^{0}\right\|_{\bar{C}} \leq \ldots \leq\left\|y_{1}^{0}\right\|_{\bar{C}}
$$

Moreover, Corollary 2.1 gives us the relationship: $0<u_{1} \leq y_{p}^{0} \leq u_{2}$, i. e., $y_{p}^{0} \in \bar{D}_{\tilde{u}}$. Now let us consider the problem $(6.5),(6.5),(4.4)$ when $n=0$. As $A_{1}^{0}>0, D_{1}^{0}=1$ and $\varphi(x, t) \geq 0$ for $(x, t) \in \bar{\omega}$, then by similar arguments, using the maximum principle and its corollaries, we obtain the estimate

$$
0<u_{1} \leq y_{1}^{1}, \quad\left\|y^{1}\right\|_{\bar{C}} \leq \max \left\{\left\|\mu^{1}\right\|_{C_{\gamma}},\left\|y^{0}\right\|_{\bar{C}}\right\}+\tau\left\|\varphi^{1}\right\|_{C}
$$

From the generalized quadrature right-rectangle formula we have the inequality

$$
\left|\sum_{k=1}^{n} \tau\left\|f^{k}\right\|_{C\left(\bar{\omega}_{h}\right)}-\int_{0}^{t}\|f(\xi)\|_{C(\Omega)} d \xi\right| \leq r
$$


Therefore, $y_{1}^{1} \in \bar{D}_{\tilde{u}}$. Now let $y_{1}^{n} \in \bar{D}_{\tilde{u}}$ and inequalities (6.1), (6.2) be satisfied for $t=t_{n}$. Then for difference equations (6.3), (6.4) it is valid the positivity conditions (2.2) for the coefficients:

$$
A_{\alpha}^{n}>0, \quad D_{\alpha}^{n}=A_{\alpha}^{n}-\sum_{\xi \in \mathcal{M}^{\prime}} B_{\alpha}^{n}(x, \xi)=1, \quad \alpha=\overline{1, p} .
$$

On the basis of the maximum principle and its corollaries we obtain the following inequalities:

$$
\begin{aligned}
0<u_{1} \leq y^{n+1} \leq\left\|y^{n+1}\right\|_{\bar{C}} \leq \max \left\{\max _{0<t \leq t_{n+1}+1}\right. & \left.\|\mu(x, t)\|_{C_{\gamma}},\left\|u_{0}\right\|_{\bar{C}}\right\} \\
& +\sum_{t^{\prime}=\tau}^{t+\tau} \tau\left\|\varphi\left(t^{\prime}\right)\right\|_{C} \leq u_{2}+r .
\end{aligned}
$$

The latter estimate means that $y^{n+1} \in \bar{D}_{\tilde{u}}$ and

$$
0<k_{1} \leq a_{\alpha}\left(x, t, y_{1}^{n+1}\right) \leq k_{2}, \quad \alpha=1,2, \ldots, p .
$$

Then the arguments are repeated. As vector-additive scheme (4.1) - (4.5) satisfies the maximum principle then it is monotone. Inequality (6.7) represents the a priori estimate of the difference scheme solution with respect to the input data of the problem. Since the problem is nonlinear, estimate (6.7), unfortunately, does not provide the classical stability of the difference scheme.

\section{REFERENCES}

[1] V. Abrashin. On a variant of alternating direction method for solving multidimensional problems of mathematical physics. I. Differents. Uravnenia, 26(2), 314 - 324, 1990. (in Russian)

[2] P. Matus. The maximum principle and some of its applications. Computational methods in applied mathematics, 2(1), $50-91,2002$.

[3] P. Matus. On stability of vector-additive schemes. Dokl. NAS of Belarus, 46(3), 64 67, 2002. (in Russian)

[4] A. Samarskii. The Theory of Difference Schemes. Marcel Dekker, Inc., New York Basel, 2001.

[5] A. Samarskii and P. Vabishchevich. Additive Schemes for Problems of Mathematical Physics. Nauka, Moskow, 1999. (in Russian)

[6] A. Samarskii, P. Vabishchevich and P. Matus. Stability of vector-additive schemes. Dokl. $R A N, \mathbf{3 6 1}(\mathbf{6}), 746$ - 748, 1998. (in Russian)

[7] P. Vabishchevich. Vector-additive difference schemes for the first order evolutionary equations. Zh. Vychisl. Mat. Mat. Fiz., 36(3), $44-51,1996$. (in Russian)

[8] S. Zaitseva and A. Zlotnik. Accuracy error estimates of vector split methods for heat conduction equation. Zh. Vychisl. Mat. Mat. Fiz., 39(3), 472-491, 1999. (in Russian)

[9] A. Zyl and P. Matus. Economical difference schemes of raised accuracy order for multidimensional parabolic equations. Zh. Vychisl. Mat. Mat. Fiz., 39(7), 1151 - 1157, 1999. (in Russian) 
Monotoninès ekonomiškos schemos kvazitiesinèms parabolinėms lygtims

N.V. Dzenisenko, A.P. Matus, P.P. Matus

Šiame straipsnyje pasiūlytas ekonomiškos vektoriškai adityvios schemos, aproksimuojančios daugiamatę kvazitiesinę parabolinę lygtị su negriežto tipo netiesiškumu. İrodyta, kad šios lygties sprendinys tenkina maksimumo principą ir todèl pasiūlytoji schema yra monotoninè. İrodymas yra pagrịstas vektoriškai-adityvios schemos ekvivalentiškumu suminès aproksimacijos schemai, kuri yra lokaliai vienmatè schema. Gauti skaitinio sprendinio aprioriniai ivverčiai maksimumo normoje. 